\title{
Quantitative, 3D Studies of the Evolution of Grain Size and Orientation in Nano- grained, Polycrystalline Thin-Films
}

\author{
A Brian Aebersold, Cécile Hébert and Duncan TL Alexander
}

Interdisciplinary Centre for Electron Microscopy (CIME), École Polytechnique Fédérale de Lausanne (EPFL), Lausanne, CH-1015, Switzerland

Polycrystalline thin-films have many technological applications, such as functional layers in thin-film devices. Typically their microstructures are determined first by an initial, dense nucleation of nano-scale crystallites with somewhat random, non-epitaxial orientations. As the film grows thicker a subsequent growth competition of these crystallites leads to rather columnar or V-shaped grains that coarsen and develop orientation texture. Cross-section TEM and 3D FIB-SEM tomography are able to provide qualitative information on the development of such microstructures. However, if we want to interpret microstructure development with respect to theoretical models or compare it to simulations, we need to make quantitative measurements of grain size and orientation with film thickness. Here, we develop a methodology pursuant to this goal, based on the example of low-pressure CVD grown polycrystalline $\mathrm{ZnO}$ thin-films used as transparent conductive oxides in thin-film solar cells [1].

Grain sizes in the samples are found down to sizes of $<10 \mathrm{~nm}$. It is clear that to be on commensurate length scales TEM-based grain measurement techniques are required, thus here we investigate the use of automated orientation mapping in the TEM using the NanoMEGAS ASTAR system [2]. While analysis of cross-section TEM samples seems an obvious choice for obtaining through-thickness orientation maps, two significant limitations are found when applied to the ASTAR system. Firstly, typical crosssection sample preparation by tripod polishing and ion beam milling produces samples that are too thick in the first $\sim 300 \mathrm{~nm}$ of film thicknesses. Essentially the smallest grains overlap in the projection through the sample. Secondly, because of the geometry of the columnar or V-shaped grains, grain boundaries are often very tilted relative to the electron beam. These two problems lead to a poor quality of orientation mapping and grain identification (Fig. 1, left). Moreover, the thin area is too limited in size to produce useful statistics on the evolution of grain size and orientation.

To produce good quality results from the ASTAR system, a geometry is needed that avoids the above three problems. Here we show that this can be done with a plan-view TEM sample geometry, made by dimpling and ion beam polishing. Firstly, sample thickness is much reduced (e.g. $t / \lambda<0.1$ for thickness $t$, inelastic scattering mean free path $\lambda$ ), so projection effects of overlapped grains are avoided; secondly, the boundaries of columnar grains are inherently more parallel with the electron beam, thus making them better defined; thirdly, a statistically important size of thin area is obtained. This leads to a much better quality of results (Fig. 2, middle).

While the above result is promising, it reduces analysis to an $x-y$ specimen plane at some ill-defined height perpendicular to the growth direction, so it is clearly not useable for the quantitative measurement of grain evolution that is the goal of the study. To resolve this problem we are pursuing the "double wedge" specimen geometry developed by Spiecker et al. [3]. Fig. 2 illustrates preliminary TEM micrographs taken of such a sample. This geometry preserves the advantages listed above of the regular plan-view TEM sample geometry for orientation mapping in TEM. However it creates this plan-view type of sample at heights that are graded through the thickness of the thin-film, from nucleation up to the 
film surface, and that can be theoretically calibrated with high accuracy. By analyzing such double wedge samples using the orientation mapping in the TEM, it will be possible to create a dataset in the $x$, $y$, and $z$ directions - i.e. in 3D - thus meeting the goal of quantitatively measuring the development of grain size and orientations during film growth. It will then be possible to resolve questions of, for instance, suspected re-nucleation during film growth, and how to precisely monitor the influence of changes in growth conditions.

References:

[1] S Faÿ et al, Thin Solid Films 518 (2010) p. 2961.

[2] EF Rauch et al, Microscopy and Analysis 22(6) (2008) p. S5.

[3] E Spiecker et al, Acta Materialia 55 (2007) p. 3521.

[4] The authors acknowledge funding from the SNSF, Grant Number 137833. L Fanni, Dr S Nicolay and Dr A Hessler-Wyser of the IMT PV-lab, EPFL are thanked for the samples and discussions.

Cross-section

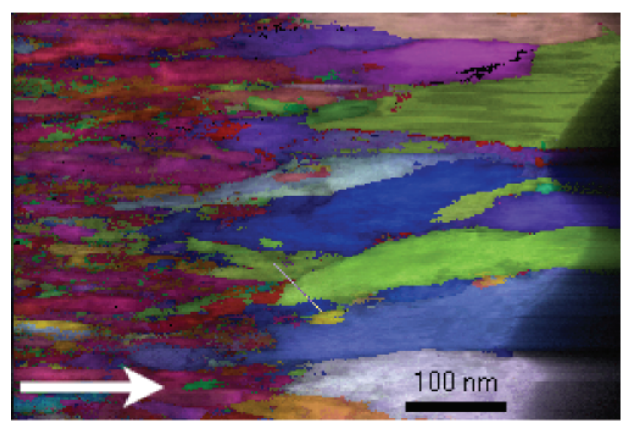

Plan-view

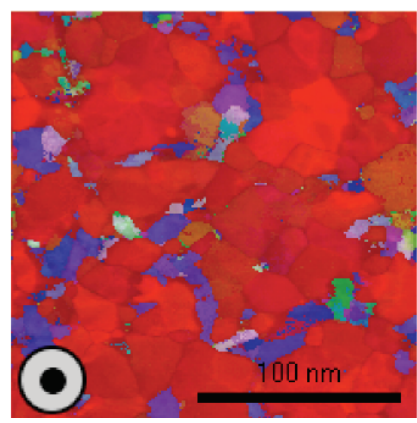

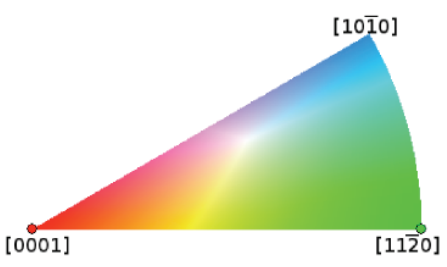

Figure 1. ASTAR map of a cross-section and a plan-view sample. The inverse pole figure shows the crystallographic direction parallel to the film normal, which is indicated by the arrows.
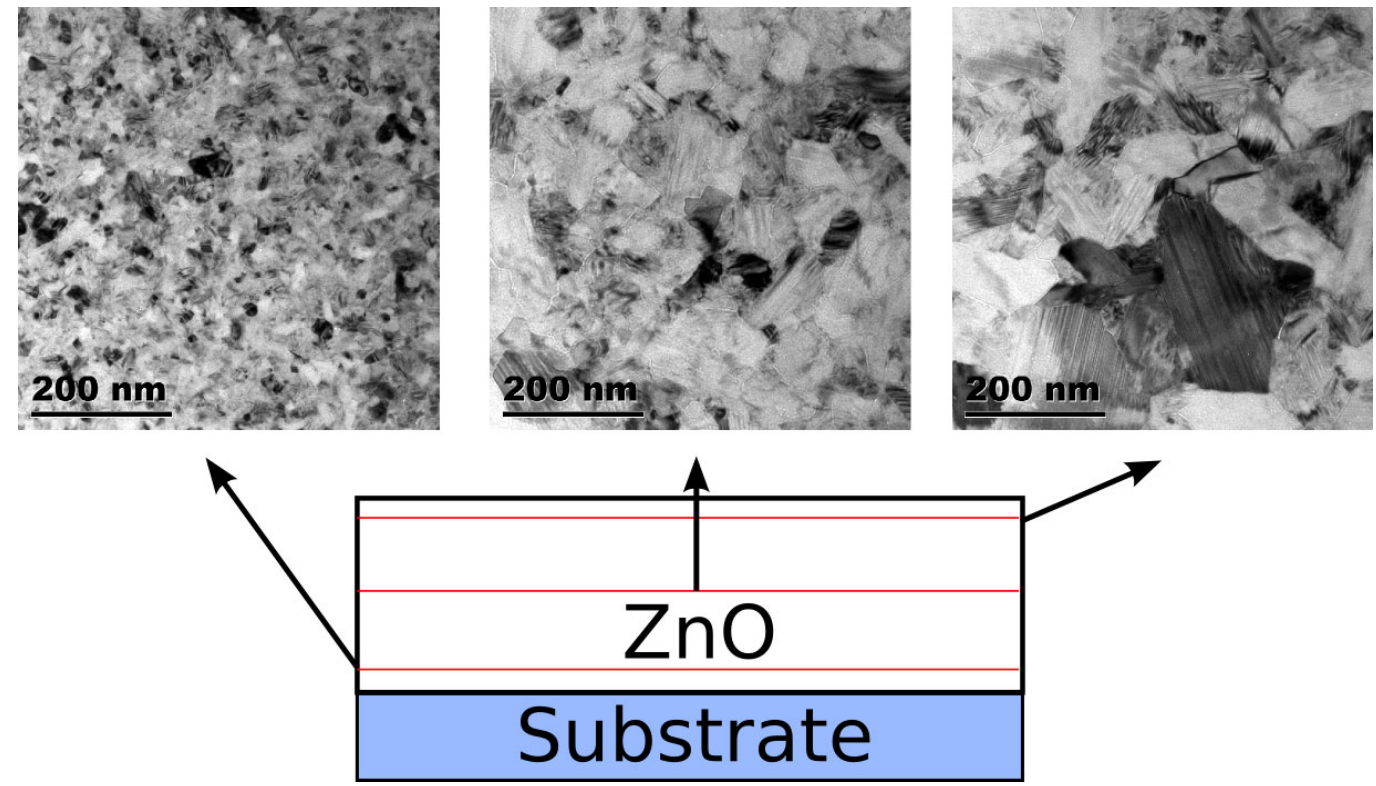

Figure 2. BF TEM images of a $\mathrm{ZnO}$ film at different heights using a "double wedge" geometry. 